\title{
Impaired bacterial clearance in IBD
}

Patients with the neurodegenerative lysosomal lipid storage disorder Niemann-Pick disease type C1 (NPC1) can present with IBD, but the functional mechanism of this association was previously unclear. New findings now shed light on this link, showing that impaired induction of autophagy resulting in defective clearance of bacteria underlies intestinal inflammation in patients with NPC1 and other genetic defects, pinpointing a potential new therapeutic target.

Polygenic IBD, in particular Crohn's disease, is associated with many genetic variants that affect bacterial clearance and autophagy. The stongest risk factor for Crohn's disease is mutations in NOD2, which is important for bacterial muramyl peptide sensing. Additionally, a number of rare monogenic disorders can also present with Crohn's disease, one of which is deficiency in XIAP, an essential signal transducer downstream of NOD2. As Crohn's disease and XIAP deficiency share a common pathway and phenotype, the researchers chose to investigate patients with NPC1, another monogenic disorder that presents with intestinal inflammation. "We think that understanding monogenic disorders with genetic defects that have high functional impact will allow us to better define key modules in the immune system that confer susceptibility to IBD in general," explains author Holm Uhlig.

Patients with NCP1 can develop granulomatous colitis, which is indicative of ineffective clearance of gut microbiota by macrophages. Thus, bacterial handling was characterized in macrophages derived from patients with NCP1 who had severe Crohn's-diseaselike intestinal inflammation with evidence of granuloma, and compared with functional defects caused by NOD2 or XIAP mutations. Using an in vitro assay of bacterial clearance, bacterial handling was first shown to be defective in NOD2-associated and $X I A P$-associated Crohn's disease, caused by impaired initiation of autophagy. Defective elimination of intracellular bacteria was then demonstrated in macrophages from patients with NCP1, which was also attributed to dysfunctional autophagy but at a critical stage of autophagosome maturation between bacterial sensing and degradation. Furthermore, pharmacological induction of autophagy was shown to restore autophagic flux and rescue in vitro bacterial handling in NPC1 macrophages.

"We think this is a very exciting finding since it links the NCP1 defect in the endosomal and lysosomal compartment with defective cytoplasmic bacterial recognition

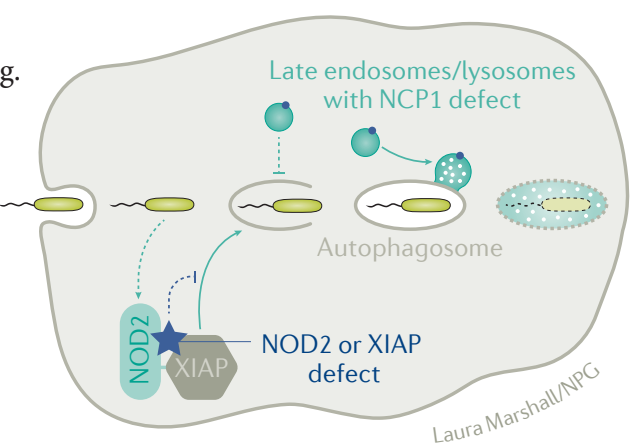

by NOD2 and signalling via XIAP. Our findings add to the concept that common and rare genetic variation in shared cellular pathways leads to shared phenotypes," says Uhlig. The effects of these heterogenous genetic defects highlight bacterial clearance as an important factor in Crohn's disease and suggest that therapeutic modification of elimination mechanisms could be a promising treatment option for IBD.

The researchers plan to investigate how other gene defects that cause Crohn's disease might affect bacterial handling and will test whether autophagy inducers can rescue immune defects in patients.

Iain Dickson

ORIGINAL ARTICLE Schwerd, T. et al. Impaired antibacterial autophagy links granulomatous intestinal inflammation in Niemann-Pick disease type $\mathrm{C} 1$ and XIAP deficiency with NOD2 variants in Crohn's disease. Gut http://dx.doi.org/10.1136/ gutjnl-2015-310382 (2016) 